\title{
Temperature and Magnetic Field Dependence of the Internal and Lattice Structures of Skyrmions by Off-Axis Electron Holography
}

\author{
K. Shibata, ${ }^{1, *}$ A. Kovács,${ }^{2}$ N. S. Kiselev,${ }^{3}$ N. Kanazawa, ${ }^{1}$ R. E. Dunin-Borkowski, ${ }^{2}$ and Y. Tokura ${ }^{1,4, \dagger}$ \\ ${ }^{1}$ Department of Applied Physics, University of Tokyo, Tokyo 113-8656, Japan \\ ${ }^{2}$ Ernst Ruska-Centre for Microscopy and Spectroscopy with Electrons and Peter Grünberg Institute, \\ Forschungszentrum Jülich GmbH, D-52425 Jülich, Germany \\ ${ }^{3}$ Institute for Advanced Simulation and Peter Grünberg Institute, Forschungszentrum Jülich GmbH, D-52425 Jülich, Germany \\ ${ }^{4}$ RIKEN Center for Emergent Matter Science (CEMS), Wako 351-0198, Japan
}

(Received 7 June 2016; published 21 February 2017)

\begin{abstract}
The internal and lattice structures of magnetic Skyrmions in B20-type FeGe are investigated using off-axis electron holography. The temperature, magnetic field, and angular dependence of the magnetic moments of individual Skyrmions are analyzed. The internal Skyrmion shape is found to vary with applied magnetic field. In contrast, the inter-Skyrmion distance remains almost unchanged in the lattice phase over the studied range of applied field. The amplitude of the local magnetic moment is found to vary with temperature, while the Skyrmion shape does not change significantly. Deviations from a circular to a hexagonal Skyrmion structure are observed in the lattice phase, in agreement with the results of micromagnetic simulations.
\end{abstract}

DOI: 10.1103/PhysRevLett.118.087202

Skyrmions are nanoscale vortexlike spin objects [1-3] that are stabilized in chiral crystals [4,5] and bilayer films [6-8] due to the Dzyaloshinskii-Moriya interaction (DMI) $[9,10]$. Skyrmions and Skyrmion lattices (SkLs) [4,5] are attracting considerable attention for their novel electrodynamic properties and potential applications [3,11]. Although spin-polarized scanning tunneling microscopy has been used to study bilayer $\mathrm{PdFe} / \operatorname{Ir}(111)$ to reveal the structures of atomic-scale SkLs [6] and the magnetic field dependence of isolated Skyrmions [8], detailed information about the SkL structure in chiral crystals remains elusive. Skyrmions and SkLs in chiral crystals can be regarded as spatially localized particlelike objects that can assemble together and are topologically protected [1,3], as confirmed by real-space observations $[5,7,8,12]$. SkLs have been described properly as multiple- $Q$ states in small-angle neutron scattering studies [13,14]. McGrouther et al. measured the magnetic field dependence of the Skyrmion internal structure using differential phase contrast imaging and showed qualitative agreement with theoretical calculations [15]. However, the quantitative real-space investigation of the dependence of Skyrmion structure on temperature $T$ and external magnetic field $B_{\text {ext }}$ is still lacking.

In this Letter, we investigate the $T$ and $B_{\text {ext }}$ dependence of the internal and lattice structures of Skyrmions using offaxis electron holography [16] in the transmission electron microscope (TEM). The technique can be used to provide real-space measurements of in-plane magnetic induction

Published by the American Physical Society under the terms of the Creative Commons Attribution 4.0 International license. Further distribution of this work must maintain attribution to the author(s) and the published article's title, journal citation, and DOI. projected onto a plane perpendicular to the incident electron beam direction $\left(\boldsymbol{k}_{\mathrm{e}}\right)$ [17]. Previously, Park et al. observed a $\mathrm{SkL}$ in $\mathrm{Fe}_{0.5} \mathrm{Co}_{0.5} \mathrm{Si}$ using off-axis electron holography and discussed its three-dimensional structure on the basis of the thickness dependence of the electron phase shift $(\phi)$ [18]. In contrast, here we focus on the spatial distribution of $\phi$ and discuss the dependence of internal Skyrmion and SkL structure on $T$ and $B_{\text {ext }}$. We examine a thin plate of B20-type $\mathrm{FeGe}$, which has suitable physical properties for electron holography measurements: a high critical temperature $T_{C}(278 \mathrm{~K})$, a relatively long periodicity of the helical magnetic order $(70 \mathrm{~nm})$, a large magnetization $\left(1 \mu_{B} / \mathrm{FeGe}\right)$ and a wide $\mathrm{SkL}$ phase region in the $T-B_{\text {ext }}$ plane for thin samples [19].

Single crystals of $B 20$-type FeGe were synthesized using a chemical vapor transport method [20]. A thin platelike sample of FeGe (110) with a thickness of approximately $120 \mathrm{~nm}$ was prepared using a focused ion beam method (NB5000, Hitachi). Off-axis electron holograms were recorded using an accelerating voltage of $300 \mathrm{kV}$ in an FEI Titan TEM equipped with a field emission electron gun, multiple electron biprisms, and an objective lens spherical aberration corrector [21]. All measurements were performed in aberration-corrected Lorentz mode with a spherical aberration coefficient $C_{\mathrm{s}}$ of below $1 \mathrm{~mm}$, which reduces image delocalization. The electron holography experiments were performed using a single biprism positioned close to one of the conjugate image planes in the microscope. The sample temperature was controlled using a liquid $\mathrm{N}_{2}$ cooling holder (Gatan model 636). An external magnetic field $\boldsymbol{B}_{\text {ext }}$ was applied parallel to the incident electron beam direction (i.e., perpendicular to the sample plane) using the magnetic field of the partially excited conventional microscope objective lens in order to stabilize Skyrmions [5]. The applied 

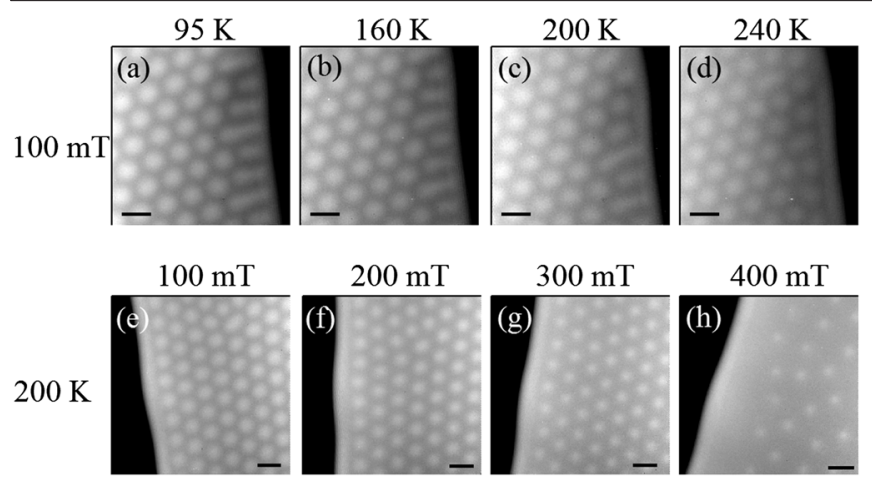

FIG. 1. Dependence on temperature $T$ and applied magnetic field $B_{\text {ext }}$ of the phase shift $(\phi)$ recorded from Skyrmions in a thin sample of FeGe using off-axis electron holography. (a)-(d) Phase shift recorded in a magnetic field of $100 \mathrm{mT}$ at a temperature of (a) 95, (b) 160, (c) 200, and (d) $240 \mathrm{~K}$. (e)-(h) Phase shift recorded at a temperature of $200 \mathrm{~K}$ in a magnetic field of (e) 100 , (f) 200, (g) 300, and (h) $400 \mathrm{mT}$. The magnetic field was always applied perpendicular to the plane of the sample $(\simeq 120 \mathrm{~nm}$ thick). All scale bars are $100 \mathrm{~nm}$.

magnetic field strength was calibrated using a Hall effect sensor positioned in a TEM holder. Real-space phase images were reconstructed from recorded holograms using HOLOWORKS software (Gatan). The structures of the observed Skyrmions were analyzed in cylindrical coordinates: $\boldsymbol{r}=(\rho \sin \varphi, \rho \cos \varphi, z)$, where the origin $(\boldsymbol{r}=\mathbf{0})$ is taken at the center of each Skyrmion, the $z$ axis is taken to be perpendicular to the film plane, and the $\varphi=0$ line is taken as the line passing through the origin and the center of a second nearest neighbor Skyrmion [see Fig. 3(b) below]. An incident electron traveling along the $+z$ direction experiences a phase shift that can be described using the equation

$$
\begin{aligned}
\phi(\rho, \varphi)= & \frac{e}{\hbar \nu} \int_{-\infty}^{+\infty} V(\rho, \varphi, z) d z \\
& -\frac{e}{\hbar} \int_{-\infty}^{+\infty} \boldsymbol{A}(\rho, \varphi, z) \cdot \boldsymbol{e}_{z} d z,
\end{aligned}
$$

where $V$ is the mean inner electrostatic potential of the sample, $\nu$ is the electron velocity, $\boldsymbol{A}$ is the magnetic vector potential, $\hbar$ is the reduced Planck constant, $e$ is the elementary electric charge, and $\boldsymbol{e}_{i}$ is a fundamental unit vector in the coordinate system $(i=\rho, \varphi, z)$ [17]. The first and second terms are the electrostatic and magnetic contributions to the phase shift, respectively.

Figure 1(a) shows a representative reconstructed phase image recorded at $95 \mathrm{~K}$ and $100 \mathrm{mT}$ after field cooling at $100 \mathrm{mT}$. The hexagonally arranged peaks in the phase image, each of which corresponds to a Skyrmion whose in-plane magnetic moments are wound counterclockwise [18], confirms the formation of a SkL even at $95 \mathrm{~K}$ $\left(\ll T_{C} \sim 278 \mathrm{~K}\right.$ ) [12]. Figures $1(\mathrm{~b})-1(\mathrm{~d})$ show the distribution of the phase $\phi$ after heating from $95 \mathrm{~K}$ in the presence of a $100 \mathrm{mT}$ magnetic field. The dependence of $\phi$ on $B_{\text {ext }}$ was also investigated. After a SkL had formed at
$200 \mathrm{~K}$ in $100 \mathrm{mT}$ by field cooling, $B_{\text {ext }}$ was increased. Figures 1(e)-1(h) show the distribution of $\phi$ measured at different $B_{\text {ext }}$ values at $200 \mathrm{~K}$. The SkL survives up to about $350 \mathrm{mT}$. The application of a $400 \mathrm{mT}$ field then annihilates some Skyrmions, while some remain [Fig. 1(h)]. At $450 \mathrm{mT}$ (not shown), no phase peaks are observed, all Skyrmions are annihilated and a ferromagnetic state is realized.

Assuming a uniform sample thickness $t$ and mean inner potential $V$, i.e., a flat and homogeneous sample, the first term in Eq. (1) takes the form of a uniform phase offset and can be ignored. The recorded phase was averaged over different Skyrmion sites and symmetrized by taking into account the sixfold and mirror symmetry of the lattice for statistical noise reduction (see Supplemental Material [22] for the averaging process and the symmetrized experimental phase maps).

We analyzed the magnetic configuration of each unit cell of the SkL using the symmetrized $\phi(\rho, \varphi)$ maps. Figure 2(a) illustrates the magnetic configuration of a Skyrmion in the SkL. The magnetic moment vectors $\boldsymbol{m}$ at the core and edge are antiparallel and parallel to the applied magnetic field $\boldsymbol{B}_{\text {ext }}=B_{\text {ext }} \boldsymbol{e}_{z}$, respectively $[4,5]$. In the intermediate region, $\boldsymbol{m}$ rotates from the core to the edge in a unique rotational sense, which is right handed in this case. We assume a cylinderlike structure, in which $\boldsymbol{m}(\boldsymbol{r})$ is homogeneous along $\boldsymbol{B}_{\text {ext }}|| \boldsymbol{e}_{z}$. This assumption does not strictly hold true, since the magnetic structure is modulated near the specimen surfaces $[24,25]$. However, such small modulations cannot significantly change the phase shift because in our micromagnetic calculations they are exponentially damped and practically vanish at a depth of $\sim 20 \mathrm{~nm}$ from the surface (see Supplemental Material [22]). As far as the unit cell of the $\mathrm{SkL}$ is concerned, it is then convenient to describe the structure as a function of distance from the center of a Skyrmion $(\rho)$, on the assumption that the Skyrmion can be treated as an almost axially symmetrical object. We compare the shapes of Skyrmions determined from different phase images by normalizing each radial phase profile $\phi(\rho)$ so that the difference $(\Delta \phi)$ between the peak and the dip is identical, as shown in Fig. 2(c) for different $T$ values at $B_{\text {ext }}=100 \mathrm{mT}$. The consistency between the curves indicates that there is no significant dependence of Skyrmion structure on $T$ over the studied range of $B_{\text {ext }}$. Figure $2(\mathrm{~g})$ shows the dependence of the normalization factor $\Delta \phi$ on $T$. The decrease in $\Delta \phi$ with increasing $T$ is attributed to a decrease in the effective magnetic moment due to thermal fluctuations. The $T$-dependent variation of $\Delta \phi$ appears to be consistent with that of the magnitude of the ordered moment [26]. Figure 2(e) shows normalized profiles of $\phi(\rho)$ plotted for different $B_{\text {ext }}$ values at $200 \mathrm{~K}$. In contrast to the absence of a $T$ dependence, the $\phi(\rho)$ profiles vary significantly with $B_{\text {ext }}$.

The spatial distribution of the direction of $\boldsymbol{m}(\boldsymbol{r})$ was used as an intuitively understandable quantity to interpret the magnetic configurations from the $\phi(\rho)$ curves. On the assumption that the thickness $t$ and mean inner potential $V$ are uniform in the sample and that there are no strong 

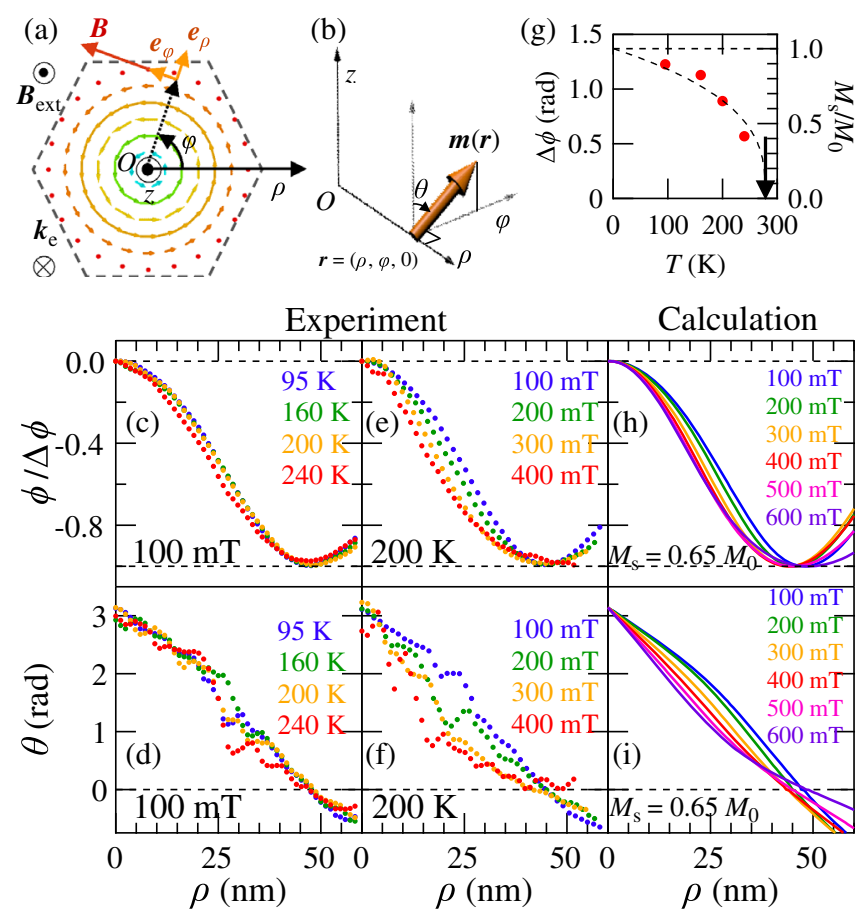

FIG. 2. Analysis of the $T$ and $B_{\text {ext }}$ dependence of internal Skyrmion structure. (a) Schematic diagram of the magnetic configuration $(\boldsymbol{m})$ of a Skyrmion, shown alongside the definition of the cylindrical coordinate system $(\rho, \varphi, z)$. $\boldsymbol{B}_{\text {ext }}$ and $\boldsymbol{k}_{\mathrm{e}}$ are the external magnetic field and the wave vector of the incident electron beam, respectively. (b) Definition of spin rotation angle $\theta$. (c) $T$ dependence of the normalized phase distribution plotted as a function of distance $(\rho)$ from the center of each Skyrmion. (d) $T$ dependence of $\theta$ plotted as a function of $\rho$. (e) and (f) show the $B_{\text {ext }}$ dependence of the normalized phase distribution and $\theta$, respectively, plotted as a function of $\rho$. $(\mathrm{g})$ Normalization factor $\Delta \phi$ for the phase curves shown in (c) plotted as a function of $T$. The right axis shows a rough estimation of $M_{s}$ in units of $M_{0}$. The dashed line is a theoretical curve for the critical exponent $\beta=0.336$ [23]. The vertical arrow indicates the magnetic transition temperature. (h) and (i) show the calculated $B_{\text {ext }}$ dependence of the normalized phase distribution and $\theta$, respectively, plotted as a function of $\rho$.

magnetic fringing fields, $\nabla \phi$ is proportional to the projected in-plane magnetic induction $\boldsymbol{B}$, which is assumed to be proportional to the projected in-plane magnetization [27]. We describe the micromagnetic calculations validating this assumption in the Supplemental Material [22]. In the present coordinate system, we assume that the relation $\nabla \phi(\boldsymbol{r})=-($ et $/ \hbar) \boldsymbol{B}(\boldsymbol{r}) \times\left(-\boldsymbol{e}_{z}\right)$ holds. In particular, we make use of the relation $(\partial \phi / \partial \rho)=\boldsymbol{e}_{\rho} \cdot \nabla \phi(\boldsymbol{r})=$ $(e t / \hbar) \boldsymbol{B}(\boldsymbol{r}) \cdot \boldsymbol{e}_{\varphi} \propto \operatorname{tm}(\boldsymbol{r}) \cdot \boldsymbol{e}_{\varphi}(\boldsymbol{r})$ along the radial $(\rho)$ direction. Assuming a fixed magnetization amplitude $M=|\boldsymbol{m}|$ and a fixed Bloch-type magnetic helicity [3] in FeGe $[19,28]$, we can describe $\boldsymbol{m}(\boldsymbol{r})$ in the form $\boldsymbol{m}=M(\sin \theta \cos \varphi, \sin \theta \sin \varphi, \cos \theta)$, where $\theta(\rho)$ is the spin rotation angle, as defined in Fig. 2(b). Then, $\theta(\rho)$ is given by the expression

$$
\begin{aligned}
\theta(\rho) & =\cos ^{-1}\left(\frac{\boldsymbol{m}(\rho) \cdot \boldsymbol{e}_{z}}{M}\right) \\
& =\cos ^{-1}\left(\operatorname{sgn}\left(\boldsymbol{m}(\rho) \cdot \boldsymbol{e}_{z}\right) \sqrt{1-\left(\frac{\partial \phi}{\partial \rho} /\left.\frac{\partial \phi}{\partial \rho}\right|_{\max }\right)^{2}}\right),
\end{aligned}
$$

where the $\operatorname{sign} \operatorname{sgn}\left(\boldsymbol{m}(\rho) \cdot \boldsymbol{e}_{z}\right)$ is taken as $1(-1)$ where $\rho>\rho_{0}\left(<\rho_{0}\right)$, and $\left.(\partial \phi / \partial \rho)\right|_{\max }$ is the maximum value of $|\partial \phi / \partial \rho|$ at $\rho=\rho_{0}$. Figures 2(d) and 2(f) show the measured $T$ and $B_{\text {ext }}$ dependence of $\theta$ plotted as a function of $\rho$, derived from Figs. 2(c) and 2(e), respectively, using Eq. (2). The value of $\rho$ at which $\boldsymbol{m}$ shows an in-plane direction $\left(\theta=\frac{\pi}{2}\right)$ becomes smaller with increasing $B_{\text {ext }}$. This behavior can be understood in terms of an increasing component of $\boldsymbol{m}(\boldsymbol{r})$ parallel to $\boldsymbol{B}_{\text {ext }}$ at large $B_{\text {ext }}$ due to a gain in Zeeman energy. Meanwhile, the inter-Skyrmion distance $a_{\mathrm{Sk}}$, which is twice the value of $\rho$ at which $\theta=-\pi$, is approximately $80 \mathrm{~nm}$ and is unaffected by $B_{\text {ext }}$, as shown in Fig. 2(f).

We compare the experimental data with the classical micromagnetic model for an isotropic chiral magnet. Equilibrium Skyrmion states are found by energy minimization of the continuum functional, which contains terms describing the Heisenberg exchange, the DMI, the Zeeman energy, and the demagnetizing field energy:

$$
\begin{aligned}
\mathcal{E}= & \int_{V_{\mathrm{s}}}\left\{\mathcal{A}\left(\partial_{x} \hat{\boldsymbol{m}}^{2}+\partial_{y} \hat{\boldsymbol{m}}^{2}+\partial_{z} \hat{\boldsymbol{m}}^{2}\right)\right. \\
& \left.+\mathcal{D} \hat{\boldsymbol{m}} \cdot[\nabla \times \hat{\boldsymbol{m}}]+M_{\mathrm{s}} \boldsymbol{B}_{\mathrm{ext}} \cdot \hat{\boldsymbol{m}}-\frac{1}{2} M_{\mathrm{s}} \boldsymbol{B}_{d} \cdot \hat{\boldsymbol{m}}\right\} d \boldsymbol{r},
\end{aligned}
$$

where $\hat{\boldsymbol{m}} \equiv \hat{\boldsymbol{m}}(x, y, z)$ is a continuous unit vector field. $\mathcal{A}$ and $\mathcal{D}$ are the micromagnetic constants for exchange interaction and DMI, respectively. $M_{s}$ is the total magnetic dipole moment per unit volume, and $\boldsymbol{B}_{d}$ is the demagnetizing field generated by the divergence of the magnetization in the sample and at its surfaces. The integration in Eq. (3) extends over the volume of the sample, $V_{s}$. We ignore the relatively weak magnetocrystalline anisotropy of $\mathrm{FeGe}$ [28] here and use the following material parameters estimated in Ref. [29]: $\mathcal{A}=8.78 \mathrm{pJ} \mathrm{m}^{-1}, \mathcal{D}=1.58 \mathrm{~mJ} \mathrm{~m}^{-2}$, and $M_{s}=384 \mathrm{kA} \mathrm{m}^{-1}$. For the correct description of the demagnetizing fields in the case of an extended film, we use periodic boundary conditions with a large number of repetitions; we take into account the demagnetizing fields produced by many copies of the simulated domain translated in the $x$ and $y$ directions [22].

We performed the calculation using MuMAx ${ }^{3}$ [30], adopting the cuboid unit cell shown in Fig. 3(a), and finding the equilibrium magnetization distribution for each value of applied magnetic field with the conjugate gradient method on a $128 \times 64 \times 64$ mesh. Since the calculated magnetic structures are almost homogeneous in the $z$ direction [22], we used the magnetization distribution averaged in this direction through the thickness of the film, $\overline{\boldsymbol{m}}(\rho, \varphi)=t^{-1} \int_{z=0}^{t} \hat{\boldsymbol{m}}(\rho, \varphi, z) d z$, for the following 

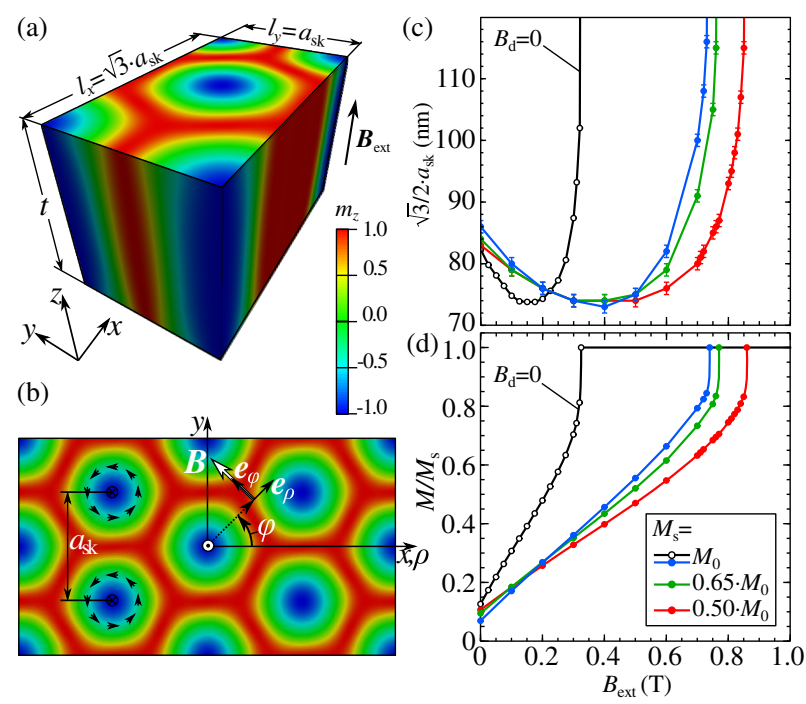

FIG. 3. Micromagnetic calculation of equilibrium SkLs for different $B_{\text {ext }}$ values. (a) Simulated domain with dimensions $l_{x}$, $l_{y}, t=100 \mathrm{~nm}$. (b) Color map of the equilibrium distribution of magnetization averaged over the $z$ direction. Cartesian $(x, y, z)$ and polar $(\rho, \varphi)$ coordinate systems used to describe the Skyrmion structure. (c) and (d) are the dependencies of the equilibrium period of the SkL and the average magnetization on $B_{\text {ext }}$, respectively. The open circles correspond to the case when magnetostatic interactions are ignored; the demagnetizing fields inside the sample are assumed to be zero, $B_{d}=0$. Colored circles correspond to equilibrium states calculated for different spontaneous magnetization, where $M_{0}$ is the spontaneous magnetization at $T=0 \mathrm{~K}$.

analyses. In order to compare the calculations with our experimental results for the $\boldsymbol{B}_{\text {ext }}$ dependence of the $\theta(\rho)$ profiles at $200 \mathrm{~K}$, we analyzed the calculation for $M_{s}=0.65 M_{0}$, considering the reduced amplitude of the magnetization due to thermal fluctuations, which is estimated from Fig. 2(g). We used polar coordinates, as shown in Fig. 3(b). The normalized $\phi-\rho$ profile $\phi(\rho)=$ $(2 \pi \rho)^{-1} \int_{\varphi=0}^{2 \pi} \int_{\rho^{\prime}=0}^{\rho} \overline{\boldsymbol{m}}\left(\rho^{\prime}, \varphi\right) \cdot \boldsymbol{e}_{\varphi} d \rho^{\prime} d \varphi$ and the calculated $\theta-\rho$ profile $\theta(\rho)=(2 \pi \rho)^{-1} \int_{\varphi=0}^{2 \pi} \cos ^{-1}\left[\overline{\boldsymbol{m}}(\rho, \varphi) \cdot \boldsymbol{e}_{z}\right] d \varphi$ are shown in Figs. 2(h) and 2(i), respectively, and provide a qualitative fit to the data deduced from the $\phi$ maps. The slight difference between the experiment and the calculation results from a systematic error due to the finite resolution of the instrument and the effect of the demagnetizing field, as discussed in Ref. [22]. Figures 3(c) and 3(d) illustrate how the equilibrium inter-Skyrmion distance $a_{\mathrm{Sk}}$ and the average magnetization change with $\boldsymbol{B}_{\mathrm{ext}}$ for different $M_{s}$ values. It is worth mentioning that the demagnetizing field significantly changes the $\boldsymbol{B}_{\text {ext }}$ dependence of $a_{\mathrm{Sk}}$ and $M_{s}$. The energy terms describing both the demagnetizing field and the Zeeman energy are sensitive to the absolute value of the spontaneous magnetization $M_{s}$ which is generally $T$ dependent. The increase in the saturation field of the SkL for reduced values of $M_{S}$ shows
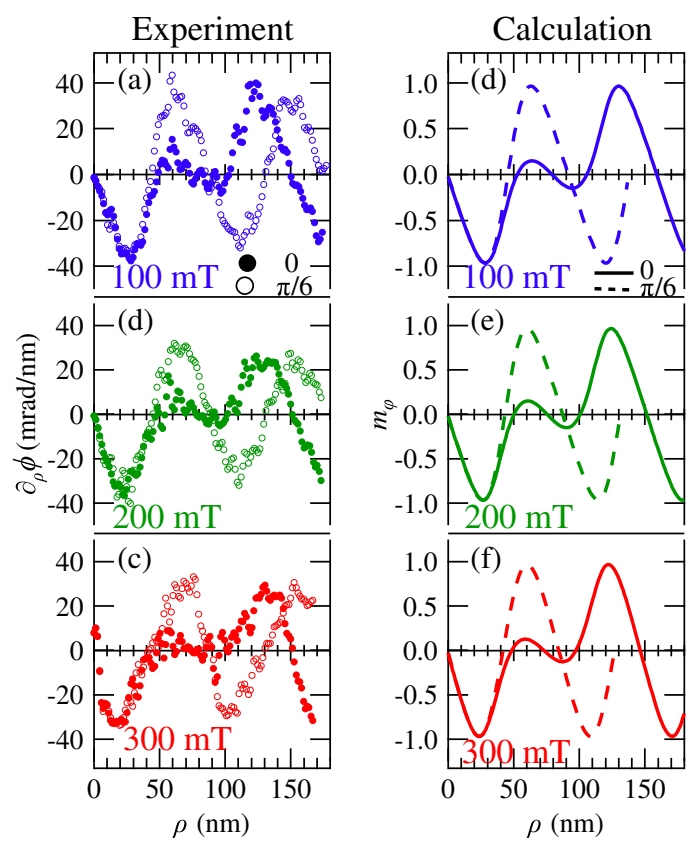

FIG. 4. Analysis of the SkL structure along the two typical directions of $\varphi=0$ and $\pi / 6$. (a)-(c) $\rho$ derivative of the phase shift $\phi$ for $B_{\text {ext }}$ values of (a) 100, (b) 200, and (c) $300 \mathrm{mT}$. (d)-(f) $\rho$ dependence of the calculated values of $m_{\varphi}$ for $B_{\text {ext }}$ of (d) 100 , (e) 200, and (f) $300 \mathrm{mT}$.

the dominant role of the Zeeman energy compared to the demagnetizing field. According to Fig. 3(c), $a_{\mathrm{Sk}}$ changes slightly from 80 to $73 \mathrm{~nm}$ as a result of the increase in external magnetic field field from 100 to $400 \mathrm{mT}$, which is consistent with the small change of $a_{\mathrm{Sk}}$ with $B_{\text {ext }}$ shown in Fig. 2(f). For magnetic fields higher than $500 \mathrm{mT}, a_{\mathrm{Sk}}$ increases very rapidly. However, at such high fields the Skyrmions become less stable with respect to shrinking and some of them collapse due to thermal fluctuations, hampering the observation of Skyrmions as a regular hexagonal lattice phase.

A further interesting question is related to the deformation of the SkL structure when the magnetic field is changed. Figures 4(a)-4(c) show $\partial \phi / \partial \rho$ calculated from the observed phase maps along two typical directions of the hexagonal SkL, $\varphi=0$ and $\pi / 6$ [see Fig. 2(a)]; $\partial \phi / \partial \rho$ represents the inplane component of $\boldsymbol{m}$ according to Eq. (2). Along the two characteristic directions, the curves cross 0 at different $\rho$ values ( $\sim 45$ and $\sim 50 \mathrm{~nm}$, respectively). This difference is associated with the deformation of a Skyrmion from a circular to a hexagonal shape in the unit cell due to the presence of surrounding Skyrmions in the SkL state. Figures 4(d)-4(f) show $m_{\varphi}=\overline{\boldsymbol{m}} \cdot \boldsymbol{e}_{\varphi}$ obtained from the micromagnetic model along the two typical directions. The shape and period in the experimental data and the calculation are in a good agreement, even without fitting.

In summary, we have investigated the internal and lattice structures of Skyrmions in a thin sample of FeGe using offaxis electron holography. Our measurement has revealed a 
dependence of the local magnetic moment on $T$, as well as an internal shrinkage of the Skyrmions with increasing $B_{\text {ext }}$. The in-plane profile of the magnetic moment in a unit cell of the SkL measured from an electron holographic phase image can be fitted well using a micromagnetic model for an isotropic chiral magnet. The in-plane profile of the magnetic moment also reveals that each Skyrmion in the lattice is deformed from a circular to a hexagonal shape. We calculated the equilibrium magnetization distribution of the Skyrmion lattice within the film to describe the experimentally observed change in the in-plane profile of the magnetic moment with applied magnetic field strength. It should be noted that, over a certain range of parameters, for a qualitative description of the Skyrmion state the effect of the stray field can be omitted; see, for example, Ref. [15]. However, as we show in this Letter, for a quantitative description it should be taken into account. Our results provide a firm basis for understanding the structures of Skyrmions and Skyrmion lattices.

The authors would like to thank X. Z. Yu, D. Morikawa, T. Kurumaji, Y. Okamura, J. Caron, and Z.-A. Li for helpful discussions. This study was supported by the Grant-in-Aid for Scientific Research (Grants No. 24224009 and No. 14J09358) from the MEXT and by the Funding Program for World-Leading Innovative R\&D on Science and Technology (FIRST Program). The research leading to these results received funding from the European Research Council under the European Union's Seventh Framework Programme (FP7/2007-2013) and under ERC Grant Agreement No. 320832. K. S. was supported by the Japan Society for the Promotion of Science through Program for Leading Graduate Schools (MERIT).

*kiyou.shibata@ riken.jp

Present address: RIKEN Center for Emergent Matter Science (CEMS), Wako 351-0198, Japan. †tokura@ riken.jp

[1] A. Bogdanov and A. Hubert, J. Magn. Magn. Mater. 138, 255 (1994).

[2] U. K. Rößler, A. A. Leonov, and A. N. Bogdanov, J. Phys. Conf. Ser. 303, 012105 (2011).

[3] N. Nagaosa and Y. Tokura, Nat. Nanotechnol. 8, 899 (2013).

[4] S. Mühlbauer, B. Binz, F. Jonietz, C. Pfleiderer, A. Rosch, A. Neubauer, R. Georgii, and P. Böni, Science 323, 915 (2009).

[5] X. Z. Yu, Y. Onose, N. Kanazawa, J. H. Park, J. H. Han, Y. Matsui, N. Nagaosa, and Y. Tokura, Nature (London) 465, 901 (2010).

[6] S. Heinze, K. von Bergmann, M. Menzel, J. Brede, A. Kubetzka, R. Wiesendanger, G. Bihlmayer, and S. Blügel, Nat. Phys. 7, 713 (2011).

[7] N. Romming, C. Hanneken, M. Menzel, J. E. Bickel, B. Wolter, K. von Bergmann, A. Kubetzka, and R. Wiesendanger, Science 341, 636 (2013).
[8] N. Romming, A. Kubetzka, C. Hanneken, K. von Bergmann, and R. Wiesendanger, Phys. Rev. Lett. 114, 177203 (2015).

[9] I. Dzyaloshinsky, J. Phys. Chem. Solids 4, 241 (1958).

[10] T. Moriya, Phys. Rev. 120, 91 (1960).

[11] J. Sampaio, V. Cros, S. Rohart, A. Thiaville, and A. Fert, Nat. Nanotechnol. 8, 839 (2013).

[12] P. Milde, D. Köhler, J. Seidel, L. M. Eng, A. Bauer, A. Chacon, J. Kindervater, S. Mühlbauer, C. Pfleiderer, S. Buhrandt, C. Schütte, and A. Rosch, Science 340, 1076 (2013).

[13] T. Adams, S. Mühlbauer, C. Pfleiderer, F. Jonietz, A. Bauer, A. Neubauer, R. Georgii, P. Böni, U. Keiderling, K. Everschor, M. Garst, and A. Rosch, Phys. Rev. Lett. 107, 217206 (2011).

[14] S. V. Grigoriev, N. M. Potapova, E. V. Moskvin, V. A. Dyadkin, C. Dewhurst, and S. V. Maleyev, JETP Lett. 100, 216 (2014).

[15] D. McGrouther, R. J. Lamb, M. Krajnak, S. McFadzean, S. McVitie, R. L. Stamps, A. O. Leonov, A. N. Bogdanov, and Y. Togawa, New J. Phys. 18, 095004 (2016).

[16] P. A. Midgley and R. E. Dunin-Borkowski, Nat. Mater. 8, 271 (2009).

[17] H. Lichte and M. Lehmann, Rep. Prog. Phys. 71, 016102 (2008).

[18] H. S. Park, X. Yu, S. Aizawa, T. Tanigaki, T. Akashi, Y. Takahashi, T. Matsuda, N. Kanazawa, Y. Onose, D. Shindo, A. Tonomura, and Y. Tokura, Nat. Nanotechnol. 9, 337 (2014).

[19] X. Z. Yu, N. Kanazawa, Y. Onose, K. Kimoto, W. Z. Zhang, S. Ishiwata, Y. Matsui, and Y. Tokura, Nat. Mater. 10, 106 (2011).

[20] M. Richardson, Acta Chem. Scand. 21, 2305 (1967).

[21] C. Boothroyd, A. Kovács, and K. Tillmann, J. Large-Scale Res. Facilities 2, A44 (2016).

[22] See Supplemental Material at http://link.aps.org/ supplemental/10.1103/PhysRevLett.118.087202 for the averaging procedure, symmetrized phase maps, and micromagnetic calculation.

[23] L. Zhang, H. Han, M. Ge, H. Du, C. Jin, W. Wei, J. Fan, C. Zhang, L. Pi, and Y. Zhang, Sci. Rep. 6, 22397 (2016).

[24] F. N. Rybakov, A. B. Borisov, and A. N. Bogdanov, Phys. Rev. B 87, 094424 (2013).

[25] A. O. Leonov, Y. Togawa, T. L. Monchesky, A. N. Bogdanov, J. Kishine, Y. Kousaka, M. Miyagawa, T. Koyama, J. Akimitsu, T. Koyama, K. Harada, S. Mori, D. McGrouther, R. Lamb, M. Krajnak, S. McVitie, R. L. Stamps, and K. Inoue, Phys. Rev. Lett. 117, 087202 (2016).

[26] B. Lebech, J. Bernhard, and T. Freltoft, J. Phys. Condens. Matter 1, 6105 (1989).

[27] R. E. Dunin-Borkowski, M. R. McCartney, D. J. Smith, and S. S. P. Parkin, Ultramicroscopy 74, 61 (1998).

[28] M. Uchida, N. Nagaosa, J. P. He, Y. Kaneko, S. Iguchi, Y. Matsui, and Y. Tokura, Phys. Rev. B 77, 184402 (2008).

[29] M. Beg, R. Carey, W. Wang, D. Cortés-Ortuño, M. Vousden, M.-A. Bisotti, M. Albert, D. Chernyshenko, O. Hovorka, R. L. Stamps, and H. Fangohr, Sci. Rep. 5, 17137 (2015).

[30] A. Vansteenkiste, J. Leliaert, M. Dvornik, M. Helsen, F. Garcia-Sanchez, and B. Van Waeyenberge, AIP Adv. 4, 107133 (2014). 